\title{
Titanium Elastic Nailing in Pediatric Femoral Diaphyseal Fractures in 6-14 Years Age Groups.
}

\author{
${ }^{1}$ Dr . M. Lakshmi Narayana, ${ }^{2}$ Dr. N .Ravi Kiran, ${ }^{3}$ Dr. G .Naresh , \\ ${ }^{1}$ Assistant Professor-Osmania Medical College-Hyderabad-Telangana. \\ ${ }^{2}$ Assistant Professor-Osmania Medical College -Hyderabad-Telangana. \\ ${ }^{3}$ Junior Resident - Osmania Medical College-Hyderabad-Telangana .
}

\begin{abstract}
Fractures of femur in pediatric age group is mainly age dependent .Treatment below 5 years mainly conservatively, by cast or traction plus casting due to good healing capacity and remodeling .Above 17years were skeletal maturity is achieved interlocking nailing is standard treatment. But in age groups 6-14 years treatment is controversial. There are number of treatment modalities likes casting, traction followed by casting external fixator, plating, elastic nailing, inter locking nailing with entry portal other than pyriform fossa. Because in this age group remodeling capacity is less and displacing muscle forces are high, requiring accurate reduction and maintenance

This is prospective study of 22 patients with single isolated femoral diaphyseal fractures in age group of 614, With transverse, short oblique and minimal comminuted fractures are treated with titanium elastic nails. results are evaluated by radiological union, weight bearing , return to school, any complications, patient satisfaction, and flynn's scoring criteria

Titanium elastic nailing is a simple operative procedure which can be done by closed method minimal incision, with minimal blood loss, less complications and high patient satisfactory rate.
\end{abstract}

Keywords: Titanium elastic nails, femoral diaphyseal fractures, pediatric age group.

\section{Introduction}

Pediatric Femoral diaphyseal fractures are2\% ${ }^{1}$ epidemiologically, they are increasing because of urbanization, motor vehicles and apartment culture. Below 5 years, patients have good union and good remodeling capacity as they have thick periosteum ,muscle displacing capacity is less, they are not school going children and patient tolerance is high. Hence can be treated with hip spica or traction followed by casting $^{2}$.

Above 17 years they are skeletally mature so can be treated rigidly by intra medullary interlocking nails. but age group of 6-16 years are not skeletally matured remodeling capacity is less, displacing forces of muscles are high and patients are school going, so accurate reduction maintenance is important in this age group and early mobilization is required ${ }^{3}$

In the past these age groups are treated by plaster immobilization, external fixator, plating and rigid intra medullary rod placing, but all have their own complications . but presently titanium elastic nailing is preferred. Titanium elastic nails are introduced $2.5 \mathrm{~cm}$ above the distal physis so that they do not interfere with the physis ,as they have good elasticity they allow micro motion at the fracture site and act as internal splint by bracing the fracture., thus converting the forces into compression forces at fracture site so that good callus is formed .They are operated with closed method with minimal incision not disturbing the fracture hematoma and periosteum ,so that early mobilization is possible ${ }^{4,5,6}$.

\section{Materials and Methods}

Patients of femoral diaphyseal fractures of 6-14 age group 22 in number are prospectively enrolled for study between November 2013 to april 2015 .only transverse, short oblique, minimum comminuted fractures Single femoral fractures with no associated injuries, within 5 days 0ld are selected. in this study there were 14 boys and 8 girls, 15are right sided and 7 are left sided, 14 are transverse and 8 are short oblique fractures .12 are middle 1/3, 6 are proximal middle $1 / 3$ junction, 4 are middle distal $1 / 3$ junction. 14 are road traffic accidents, 6 are fall from a height, 2 fell down while playing.

Exclusion criteria.

a) below 5 years

b) above 14 years

c) spiral fractures

d) comminuted

e) associated injuries

f) end fractures 
g) more than 5 days old fractures

h) compound fractures

\section{III. operative procedure}

After investigations and fitness taken patients were posted for surgery. under general anesthesia on fracture table or plain table, an incision taken on the medial side 2.5 to $3.5 \mathrm{~cm}$ above the distal physis and $2.5 \mathrm{~cm}$ distally, muscle is split. entry is in the middle of the bone with awl or drill bit $2.5 \mathrm{~cm}$ above the distal physis firstly at $90^{\circ}$ slowly tilting to $45^{\circ}$ proceed into the bone two nails of suitable diameter are selected by using Flynn's formula, the narrowest diameter of the bone in Ap and lateral views multiplied by 0.40. nail is bent to c -shaped with maximum convexity corresponding to fracture site, nail of corresponding diameter is entered in entry hole with the $\mathrm{T}$ handle or inserter, by slowly taping up to fracture site. another entry laterally $2.5 \mathrm{~cm}$ above the distal physis is done. Another nail of same diameter is inserted up to fracture site and fracture is reduced. the nail which reduces the fracture easily is introduced first followed by second one. The lateral side nail tip should be $1 \mathrm{~cm}$ away from greater trochanter and medial side nail should be $2 \mathrm{~cm}$ away from capital physis the nail maximum convexity at fracture site, with the two nails of same diameter they will now brace the fracture internally giving maximum stability. now confirm the fracture reduction and check for the proximal nail tips and confirm their proper positions. Mark on the nail $1 \mathrm{~cm}$ away from entry site for cutting. retracting the nails outside cut the nails and reintroduce them to their respective positions. bend the cut end of the nail $10^{\circ}$ away from the bone.

Postoperatively above knee slab applied depending on the stability of the fracture. limb elevation done, patient is immobilized in slab for two weeks ,slab is removed after two weeks and mobilization started . after 4 weeks pains is decreased . after $\mathrm{X}$-ray is checked partial weight bearing started at $6^{\text {th }}$ week full weight bearing started .

\section{Results}

All the 22 fractures were healed, and are available for evaluation ,they were followed up for one year. surgery time was 60-90 minutes, blood loss was of less than50 ml ,hospital stay was for 5-7 days ,partial weight bearing was started in 4-6 weeks, full weight bearing was started in 6-10weeks,radiological union was achieved in 6-10 weeks, they got full knee movements within 12 weeks, started to go to school with in 10 weeks.

In this study there were two superficial infections at entry site, which were healed after a course of antibiotics, limb lengthening was below $1 \mathrm{~cm}$ in two patients, mild complications of bursitis at entry site were two, below $10^{\circ}$ angulations were four, among them two were varus two were valgus ,scoring by flynn's critirea $16(7 \mathrm{o} \%)$ were excellent, $8(30 \%)$ were good and no poor outcomes were seen

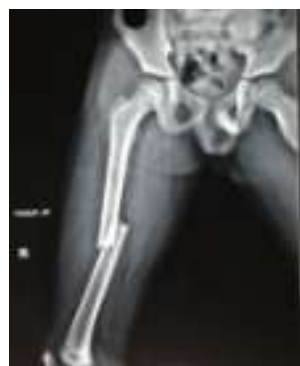

Preop x ray

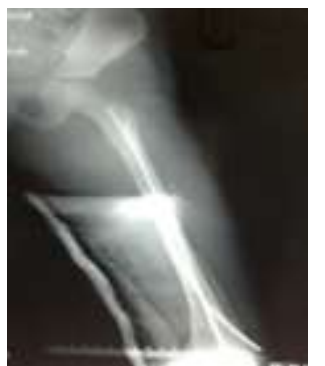

immediate postop

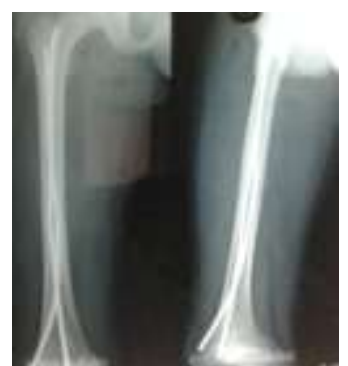

follow up x ray

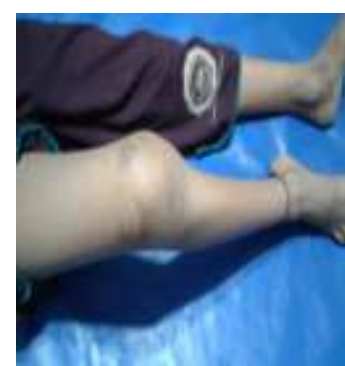

good healed minimal incision scar

Pediatric femoral fractures are in an increasing trend, because of increased urbanization, road traffic accidents , apartment culture. The age group of 6-16 years have poor remodeling capacity, high displacing muscle forces, and are school going age, requires accurate reduction, maintenance of reduction and early mobilization with less complications, in view of skeletal immaturity and patient friendly management .

In the Past these age groups were treated by traction and casting, but prolonged immobilization ,poor maintainace of reduction, leading to many complications, patient intolerance and psycho social economic impact on the family ${ }^{7,10,11}$.

Plating is another alternative but requires long incision, loose fracture hematoma, requires periosteal stripping, more blood loss, that leads to more infections, delayed unions, more immobilization, increased patient morbidity and also requires long incision for removal, and refractures after plate removal ${ }^{9}$.

External fixator is another alternative. it is present on the body, which is cumbersome, Maintenance difficulty, pin tract infections, malunions ,more refractures , and prolonged immobilization ${ }^{8}$. Enders rods are also an alternative, but their elastic modulus is high, uncompatable to mri and fracture deformation is high ${ }^{12}$.

Titanium elastic nails have good elasticity, easy to introduce, and they brace the fracture internally well because of its good elastic recoil capacity, fracture maintenance is also good, and has least reaction with 
the body. procedure is simple because of small incision and, fracture hematoma, periosteum and physis are not disturbed, Cosmetically is good . early mobilization is also possible. and thus it is a cost effective and patient friendly ${ }^{7,10}$.

In our study of 22 patients, surgery time was 60-90 minutes, blood loss was $50 \mathrm{ml}$,hospital stay was 57 days, full weight bearing and radiological union was by 6-8 weeks, return to school was within 10 weeks, with very few complications like Two superficial infections which were completely treated with course of antibiotics, limb lengthening less than $1 \mathrm{~cm}$ in two ,two bursitis , four below $10^{10}$ degree angulation. $70 \%$ results were excellent ,30\% were good. but there were no poor results as scored by Flynn's criteria.

In studies like john Flynn ${ }^{3}$ et al, $\mathrm{kc}_{\mathrm{s}}$ saikia $^{4}$ et al ,r $\operatorname{singh}^{6}$ et al, saseedhar ${ }^{5}$ et al, have achieved good results with titanium elastic nailing compared to casting and traction . M. iqbal ${ }^{8}$ et al achieved good results with titanium elastic nailing compared to external fixator .C. aksoy ${ }^{9}$ et al achieved good results compared to plating Narayanan etal stated that transverse short oblique minimal comminuation fractures are good for titanium elastic nailing.

\section{Conclusion}

pediatric femoral diaphyseal fractures in the age group of 6-14years Titanium elastic nailing is the best treatment option in view of good clinical ,radiological, cost effective results, high patient and parent acceptance rates. The operative procedure being simple, with minimal incision, less blood loss, no effect on the physis and fracture hematoma, and no periosteal stripping . selection of the patients and strictly adhering to the principles and technique, makes this the best treatment option in this age group .

\section{References}

[1]. Hinton RY, Lincoln A, Crockett MM, Sponseller P, Smith G (1999) Fractures of the femoral shaft in children. Incidence, mechanisms, and sociodemographic risk factors. J Bone Joint Surg Am 81:500-5092.

[2]. Buckley SL. Current trends in the treatment of femoral shaft fractures in children and adolescents. Clin Orthop Relat Res. 1997;338:60-73

[3]. Flynn, John M., et al. "The operative management of pediatric fractures of the lower extremity." J Bone Joint Surg Am 84.12 (2002): 2288-2300.

[4]. Saikia, K. C., et al. "Titanium elastic nailing in femoral diaphyseal fractures of children in 6-16 years of age." Indian journal of orthopaedics 41.4 (2007): 381.

[5]. Saseendar, S., J. Menon, and D. K. Patro. "Treatment of femoral fractures in children: is titanium elastic nailing an improvement over hip spica casting?."Journal of children's orthopaedics 4.3 (2010): 245-251.

[6]. Singh, Roop, et al. "Titanium elastic nailing in pediatric femoral diaphyseal fractures." Indian Journal of Orthopaedics 40.1 (2006): 29.

[7]. El-Adl, Gamal, et al. "Titanium elastic nail fixation for paediatric femoral and tibial fractures." Acta Orthopædica Belgica 75.4 (2009): 512.

[8]. Iqbal, Mohammad, et al. "Comparative Study of Fracture Shaft of Femur in Children Treated with Titanium Elastic Nail and Early External Fixator."Annals of King Edward Medical University 16.2 (2010).

[9]. Aksoy C, Çaolar Ö, Yazycy M, Surat A. PEDIATRIC FEMORAL FRACTURES: A COMPARISON OF COMPRESSIONPLATE FIXATION AND FLEXIBLE INTRAMEDULLARY NAIL FIXATION. InOrthopaedic Proceedings 2003 Mar 1 (Vol. 85 , No. SUPP III, pp. 263-263). Orthopaedic Proceedin

[10]. Gaid, Moheb, and Parminder Jeer. "Cost analysis of managing paediatric femoral shaft fractures: flexible intramedullary nailing versus non-operative management." Acta orthopaedica belgica 72.2 (2006): 170.

[11]. Metaizeau, J-P. "Stable elastic intramedullary nailing for fractures of the femur in children." Bone \& Joint Journal 86.7 (2004): $954-$ 95

[12]. Mahar AT, Lee SS, Lalonde FD, Impelluso T, Newton PO. Biomechanical comparison of stainless steel and titanium nails for fixation of simulated femoral fractures. J Pediatr Orthop.2004; 24: 638-41.24638 2004. 